\title{
Influence of the dielectric substrate on the terahertz electric near-field of a hole in a metal
}

\author{
L. Guestin ${ }^{1}$, A. J. L. Adam ${ }^{1 *}$, J. R. Knab ${ }^{1}$, M. Nagel $^{2}$ \\ and P. C. M. Planken ${ }^{1}$ \\ ${ }^{1}$ Delft University of Technology, Faculty of Applied Sciences, \\ Department of Imaging Science and Technology, Optics Research Group \\ Lorentzweg 1, 2628 CJ Delft, The Netherlands \\ ${ }^{2}$ Institut für Halbleitertechnik, RWTH Aachen University, \\ Sommerfeldstr. 24, 52074 Aachen, Germany \\ *Corresponding author: a.j.l.adam@tudelft.nl
}

\begin{abstract}
We have studied theoretically and experimentally the influence of a dielectric substrate on the frequency-dependent terahertz electric near-field of a small hole in a metal layer. We find that the near-field transmission spectrum and the two-dimensional field distribution of an empty hole in a thin metal layer on a substrate are almost identical to that of a hole which is also filled with the same dielectric material as the substrate. For thicker metal layers, however, the near-field spectra of filled and unfilled holes become very different. In addition, for thick metal layers, the two-dimensional field distributions are more strongly affected by the substrate, especially if we allow for an air gap between the metal and the substrate. Our results validate the -somewhat unusual- two-dimensional field distribution measured beneath a hole in a thick metal foil and highlight the effect that a substrate can have on the measurement of the near-field of an object.
\end{abstract}

(C) 2009 Optical Society of America

OCIS codes: (110.6795) Terahertz imaging; (050.6624) Subwavelength structures, (050.1220) Apertures.

\section{References and links}

1. T. W. Ebbesen, H. J. Lezec, H. F. Ghaemi, T. Thio and P. A. Wolff, "Extraordinary optical transmission through sub-wavelength hole arrays," Nature (London) 391, 667-669 (1998).

2. C. Genet and T. W. Ebbesen, "Light in tiny holes," Nature (London) 445, 39-46 (2007).

3. R. Gordon, D. Sinton, K. L. Kavanagh and A. G. Brolo, "A New Generation of Sensors Based on Extraordinary Optical Transmission," Accounts of Chemical Research 41, 1049-1057 (2008).

4. M. Beruete, M. Sorolla, I. Campillo, J. S. Dolado, L. Martn-Moreno, J. Bravo-Abad, and F. J. Garca-Vidal, "Enhanced millimeter-wave transmission through subwavelength hole arrays," Opt. Lett. 29, 2500-2502 (2004).

5. T. Matsui, A. Agrawal, A. Nahata, and Z. V. Vardeny, "Transmission resonances through aperiodic arrays of subwavelength apertures," Nature (London) 446, 517-521 (2007).

6. M. A. Seo, A. J. L. Adam, J. H. Kang, J. W. Lee, S. C. Jeoung, Q. H. Park, P. C. M. Planken, and D. S. Kim, "Fourier-transform terahertz near-eld imaging of one-dimensional slit arrays: mapping of electric field, magnetic field, and Poynting vectors," Opt. Express 15, 11781-11789 (2007).

7. O. Mitrofanov, L. N. Pfeiffer, and K. W. West, "Generation of low-frequency components due to phase-amplitude modulation of sub-cycle far-infrared pulses in the near-field diffraction," Appl. Phys. Lett. 81, 1579-1581 (2002).

8. M. van Exter and D. R. Grischkowsky, "Characterization of an Optoelectronic Terahertz Beam System," IEEE Trans. Microwave Theory Tech. 38, 1684-1691 (1990). 
9. A. J. L. Adam, J. M. Brok, M. A. Seo, K. J. Ahn, D. S. Kim, J. H. Kang, Q. H. Park, M. Nagel, and P. C. M. Planken, "Advanced terahertz electric near-field measurements at sub-wavelength diameter metallic apertures," Opt. Express 16, 7407-7417 (2008).

10. C. J. Bouwkamp "On Bethe's theory of diffraction by small holes," Philips Research Reports, 5, $321-332$ (1950).

11. F. Garcia de Abajo, "Light transmission through a single cylindrical hole in a metallic film," Opt. Express 10, 1475-1484 (2002).

12. G. Zhao, R. N. Schouten, N. C. J. van der Valk, and P. C. M. Planken, "Design and performance of a THz emission and detection setup based on a semi-insulating GaAs emitter," Rev. Sci. Instrum. 73, 1715-1719 (2002).

13. A. J. L. Adam, J. M. Brok, P. C. M. Planken, M. A. Seo, and D. S. Kim, "THz near-field measurements of metal structures," C.R. Physique 9, 161-168 (2008).

14. A. Bitzer and M. Walther, "Terahertz near-field imaging of metallic subwavelength holes and hole arrays," Appl. Phys. Lett. 92, (7) 231101 (2008).

15. A. Bitzer, H. Merbold, A. Thoman, T. Feurer, H. Helm, and M. Walther, "Terahertz near-field imaging of electric and magnetic resonances of a planar metamaterial," Opt. Express 17, 3826-3834 (2009).

16. Field Theory of Guided Waves, R. E. Collin, 2nd Edition, Publisher (Oxford University Press, USA, 1996).

17. J. R. Knab, A. J. L. Adam, M. Nagel, E. Shaner, M. A. Seo, D. S. Kim, and P. C. M. Planken, ’Terahertz near-field vectorial imaging of subwavelength apertures and aperture arrays," Opt. Express 17, 15072-15086 (2009).

\section{Introduction}

In the past decade, the study of light transmission by holes and hole arrays in metal films has led to many discoveries and applications.[1, 2,3] Although most of the work has concentrated on visible and near-infrared light or in the millimeter wave range [4], recent experiments have also been performed at terahertz (THz) frequencies. [5, 6, 7] One advantage of using THz light is that techniques exist, such as terahertz time-domain spectroscopy (THz-TDS) [8], in which the full, time-dependent electric field of a broadband, single-cycle light pulse is detected, rather than the light intensity. In the THz frequency range, the skin depth of light in a metal such as gold is on the order of $80 \mathrm{~nm}$, which is not very different from the skin depth of visible light. To study light transmission by holes and hole arrays in metal films therefore, it suffices to use metal layers which are hundreds of nanometers thick to prevent direct transmission of light through the metal. Metal layers of such a small thickness, however, require a substrate to support them, leading to the question how the substrate influences the transmission properties of the holes.

Recently, we reported on the measurement of the $\mathrm{THz}$ electric near-field of sub-wavelength-sized holes fabricated in a $200 \mathrm{~nm}$ thick gold film deposited on a GaP crystal.[9] In these experiments, we measured the frequency-dependent transmission of a hole in a plane immediately beneath it, allowing us to observe details in the electric near-field with a spatial resolution up to two orders of magnitude smaller than the diameter of the hole. At low $\mathrm{THz}$ frequencies, when the wavelength is much larger than the hole diameter, we were able to make a detailed comparison between our measurements and the predictions made by Bouwkamp [10] for the transmission of light by small sub-wavelength sized holes. Our experimental results also indicated that the strongest $\mathrm{THz}$ electric fields occurred at a frequency close to the calculated cut-off frequency of a circular waveguide, assuming that this waveguide is entirely filled with the dielectric material on which the metal is deposited. In fact, the hole, although having a depth of only $200 \mathrm{~nm}$ is not filled with GaP. Intuitively, it is to be expected that the substrate plays an important role in the transmission properties of the hole in the metal layer. However, the interplay between hole diameter, metal layer thickness, and presence of the substrate, is currently, as far as we know, not well understood.

Here, we provide measurements and calculations of the effect of the dielectric substrate on the frequency-dependent $\mathrm{THz}$ electric near-field. Our calculations show that for a subwavelength, circular hole on $\mathrm{GaP}$ having a diameter of $100 \mu \mathrm{m}$, typical of the hole sizes studied in our experiments, the transmission properties of the unfilled hole and the same hole also filled with $\mathrm{GaP}$ are virtually identical. This is true only if the thickness of the metal layer deposited on the GaP substrate is less than $2 \mu \mathrm{m}$. Measurements of the in-plane electric

\#114714 - \$15.00 USD Received 23 Jul 2009; revised 11 Sep 2009; accepted 11 Sep 2009; published 15 Sep 2009

(C) 2009 OSA

28 September 2009 / Vol. 17, No. 20 / OPTICS EXPRESS 17413 
near-field component, $E_{x}$, and the longitudinal component, $E_{z}$, beneath a $100 \mu \mathrm{m}$ diameter hole fabricated in a $200 \mathrm{~nm}$ thick gold layer deposited on $\mathrm{GaP}$ confirm our calculations. When the metal layer thickness increases, our calculations predict that the frequency-dependent transmission of both filled and unfilled holes on GaP changes dramatically. The differences in the frequency-dependent transmission between filled and unfilled holes become substantial, even for a metal layer thickness of only $5 \mu \mathrm{m}$, which is still only a small fraction of the hole diameter. At the same time, at frequencies below the lowest cut-off frequencies, the two-dimensional electric field patterns calculated in a plane beneath the hole are very similar, showing mostly changes in amplitude. For metal foils of $200 \mu \mathrm{m}$ thickness, calculations show that the influence of the substrate on the electric near-field spectrum beneath the metal foils is relatively limited. We find that this agrees with measurements of $E_{x}$ and $E_{z}$, which show cut-off frequencies corresponding to the ones of free-standing foils. However, the spatial distribution of the amplitude of the electric near-field is more perturbed by the presence of the GaP than in the case of the thin layer. For thick metal foil, we also study the effect of the presence of an air gap between the metal and the crystal. These calculations show fields that extend to regions under the metal at a considerable in-plane distance from the hole. Our results provide important information on how the electric near-field of a sub-wavelength hole is affected by the presence of the substrate, as well as the influence of the metal layer thickness on the frequency-dependent transmission of such a hole.

\section{Thin metal layer: influence of the GaP crystal}

\subsection{Frequency dependence}

In order to understand the influence of the GaP crystal underneath our structure, we have calculated, using CST Microwave Studio, the electric near-field for three configurations as shown in Fig. 1. In all cases, a $100 \mu \mathrm{m}$ diameter hole is defined in a $0.5 \mu \mathrm{m}$ thick gold layer, that is assumed to be a perfect conductor. The electric field of the $\mathrm{THz}$ pulse is incident on the hole from above and linearly polarised along the $x$ direction. The first configuration assumes that the metal layer is free standing. For the second configuration, we add a GaP crystal in contact with the bottom of the metal layer. The $\mathrm{THz}$ index of refraction is taken equal to 3.4. The last configuration is similar to the previous one, except that, in addition, the hole is now also filled with the same GaP material. These three cases will be referred to later as "Free standing", "In contact" and "In contact and filled", respectively.

In Figs. 2 (a) and (b), we plot the electric near-fields, $\left|E_{x}\right|$ and $\left|E_{z}\right|$, calculated at a fixed position in a plane $10 \mu \mathrm{m}$ below the hole, as a function of frequency, for each of the three configurations shown in Fig. 1. For $\left|E_{x}\right|$, a position exactly under the center of the aperture was chosen; for $\left|E_{z}\right|$, a position underneath the edge was chosen, where this field component is strongest. The near-field spectra are normalized with respect to the input spectrum of the incident pulse.

For $\left|E_{x}\right|$, the calculations show that for the free-standing film, the amplitude first increases, then reaches a maximum around $1.4 \mathrm{THz}$, before finally decreasing again. These results for the individual fields resemble the results obtained by Garcia de Abajo [11] for the overall transmitted power of a free standing hole. In the "In contact" and "In contact and filled" cases, the maximum is less pronounced and looks more like a plateau from $0.5 \mathrm{THz}$ till $1.5 \mathrm{THz}$. A striking result is that the curves calculated in the "In contact" and "In contact and filled" cases do not differ significantly from each other.

The calculated $\left|E_{z}\right|$ shows a similar behavior. The amplitude in the "In contact" and "In contact and filled" cases slowly increases with frequency until it reaches a maximum around $0.6 \mathrm{THz}$, followed by a slow decay. In the "Free standing" case, the maximum is reached at a much higher frequency, around $1.4 \mathrm{THz}$, which is similar to that observed for $\left|E_{x}\right|$. Here too,

\#114714 - \$15.00 USD Received 23 Jul 2009; revised 11 Sep 2009; accepted 11 Sep 2009; published 15 Sep 2009

(C) 2009 OSA

28 September 2009 / Vol. 17, No. 20 / OPTICS EXPRESS 17414 

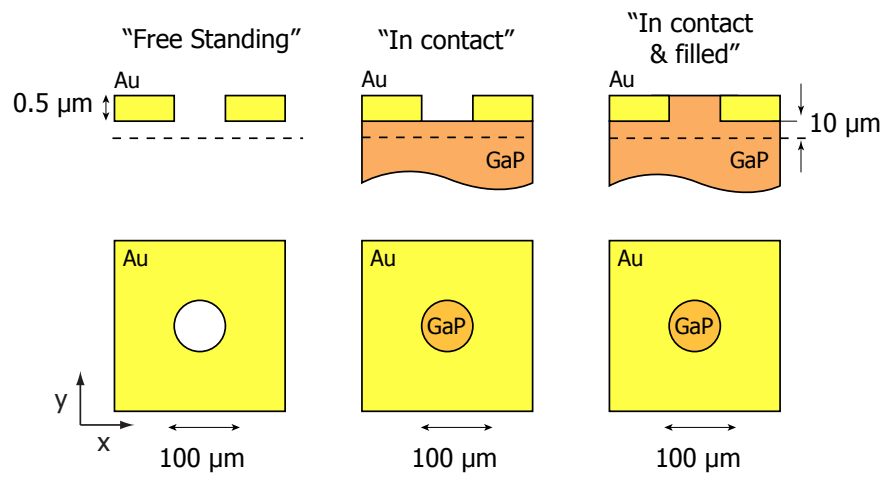

Fig. 1. Drawing of the three different configurations used in the calculations of the electric near-field under a thin metal layer containing a hole. The terahertz pulse is incident from above and polarised along the $x$-axis. In the three cases, the circular hole has a diameter of $100 \mu \mathrm{m}$. The thickness of the gold layer is $0.5 \mu \mathrm{m}$. All fields were calculated in a plane $10 \mu \mathrm{m}$ below the metal layer. Left: the metal is free standing. Middle: a layer of GaP, index $\mathrm{n}=3.4$, is placed in contact with the metal layer. Right: the GaP crystal is in contact with the metal layer and also fills the hole.

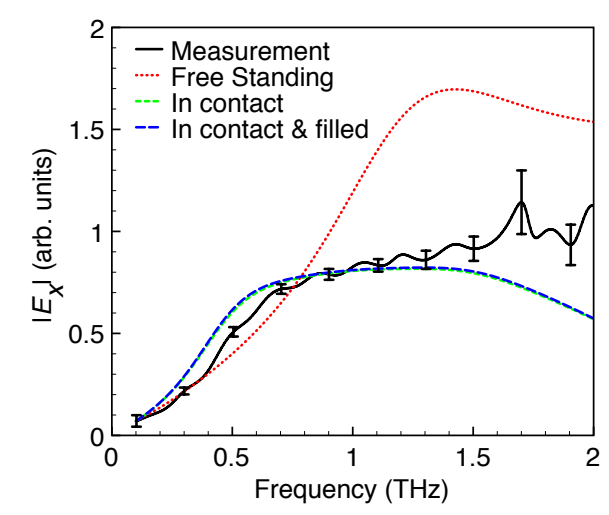

a)

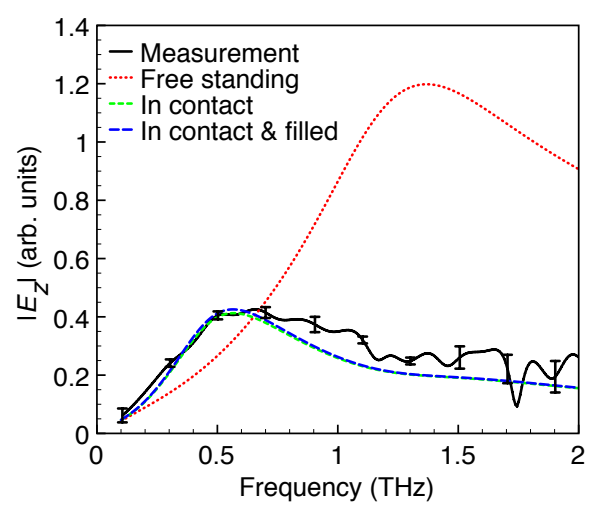

b)

Fig. 2. Calculated electric field component a) $\left|E_{x}\right|$ and b) $\left|E_{z}\right|$ as a function of frequency, $10 \mu \mathrm{m}$ below a $100 \mu \mathrm{m}$ diameter hole made in a $0.5 \mu \mathrm{m}$ thick metal layer. The red dotted line corresponds to the "Free standing" case. The broken lines correspond to the configuration in which the $\mathrm{GaP}$ is in contact with the metal and fills the hole (see Fig. 1). The solid line is the data from a measurement performed on a $100 \mu \mathrm{m}$ diameter hole made in a $200 \mathrm{~nm}$ thick gold layer which is directly deposited onto a GaP crystal. All near-field spectra are normalized with respect to the spectrum of the incident pulse. 
we observe that the curves in the "In contact" and "In contact and filled" cases, are practically indistinguishable.

We now wish to compare the calculations with actual measurements of the near-field components. For this we use a near-field setup, capable of measuring all three components, $E_{x}, E_{y}$, and $E_{z}$, of the electric near-field underneath the hole with a spatial resolution of about $10 \mu \mathrm{m}$. The setup is described in detail elsewhere $[12,13]$ and is distinct from other setups used to measure the near-field in that the longitudinal field component is also measured[14, 15].
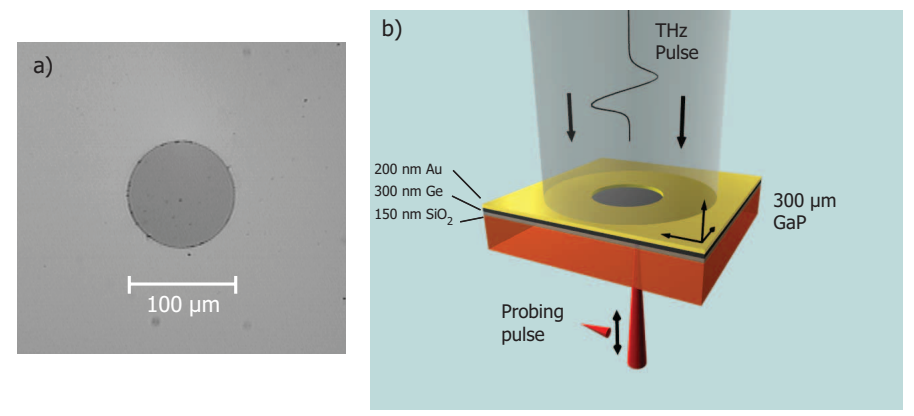

Fig. 3. a) Optical image of the $100 \mu \mathrm{m}$ hole made in a $200 \mathrm{~nm}$ thick layer of gold deposited on the $300 \mu \mathrm{m}$ thick GaP crystal. b) Detail of the experimental setup to measure the $z$-component of the near-field of a hole. The electric field of a $\mathrm{THz}$ pulse is incident on the hole from above. The local electric field $E_{z}$ is measured using the synchronized probe laser pulse (red). A highly reflective combination of a Germanium $(\mathrm{Ge})$ and $\mathrm{a} \mathrm{SiO}_{2}$ layer, prevents the probe from reaching the gold layer.

The sample consists of a $100 \mu \mathrm{m}$ diameter hole fabricated in a $200 \mathrm{~nm}$ thick gold film deposited on top of a $300 \mu \mathrm{m}$ thick GaP electro-optic detection crystal (Fig. 3 (a)). A broadband, quasi-single cycle $\mathrm{THz}$ pulse is incident on the hole. A focused probe pulse is used to sample the electric field on the shadow side of the hole, in a plane approximately $10 \mu \mathrm{m}$ below the metal inside the GaP electro-optic detection crystal (Fig. 3 (b)).[9] In Figs. 2 (a) and (b), we plot the resulting electric near-field spectra of $\left|E_{x}\right|$ and $\left|E_{z}\right|$, measured at the same locations as those used in the calculations, namely underneath the center of the hole and underneath the edge, respectively. The error bars represent the deviation of the amplitude observed at different pixel locations that are close to each other. A vertical scaling factor was applied to the measurements in order to highlight their similarity with the calculations for the "In contact" and "In contact and filled" cases. Overall, the measurements are in good agreement with the calculations, except above those frequencies where the signal-to-noise in the experiment decreases due to the decreasing amplitude of the incident broadband $\mathrm{THz}$ pulse at those frequencies.

As we will discuss below, for thicker metal layers, a clear cut-off frequency is observed, below which very little light is transmitted by the hole. For thin metal layers, however, the cut-off is not abrupt, and a significant transmission below the cut-off frequency of the hole is observed. In order to extract a cut-off frequency from our measurements and calculations of both cases, we define the "cut-off" as the frequency for which the amplitude spectrum is maximum or becomes plateau-like. We observe a "cut-off" frequency around $0.5 \mathrm{THz}$ in both the measurements and the calculations, the latter in the "In contact" and "In contact and filled" cases. In the "Free standing" case, the "cut-off" in the calculations is found at a much higher frequency, around $1.4 \mathrm{THz}$, again emphasizing the effect of the substrate on the electric near-field of the hole.

\#114714 - \$15.00 USD Received 23 Jul 2009; revised 11 Sep 2009; accepted 11 Sep 2009; published 15 Sep 2009 
Note also that the electric field under the free standing layer is stronger in amplitude than the field calculated underneath the holes on GaP. This can be understood from an increased reflection of the incident $\mathrm{THz}$ pulse due to the presence of the crystal.

We can conclude from these results that a small hole in a thin metal layer on top of GaP behaves almost exactly as a hole in a thin metal layer on top of GaP, with the hole also filled with GaP. As we will show below, this is only true for very thin metal layers.

\subsection{Near-field distribution}

In the previous section, we showed how the spectral response of the hole is affected by the presence of the substrate. To determine whether the two-dimensional field distribution underneath the hole is also changed by the substrate, we plot in Fig. 4 the amplitude of the three components of the electric near-field, $\left|E_{x}\right|,\left|E_{y}\right|,\left|E_{z}\right|$, under a $100 \mu \mathrm{m}$ diameter hole at $0.3 \mathrm{THz}$. This frequency corresponds to a free space wavelength of $1 \mathrm{~mm}, 10$ times larger than the hole diameter. Note that this frequency is lower than the cut-off frequencies of the holes for all configurations. The images in the top row represent the measured field, the lower rows correspond to the electric near-field components, calculated in a plane $10 \mu \mathrm{m}$ below the metal layer for the three configurations shown in Fig. 1. They correspond to the "Free standing", "In contact" and "In contact and filled" cases. In the calculations for the three configurations, the same color scale was used for each field-component separately. This allows for a direct comparison between the results of the calculations of, for example, the various $\left|E_{y}\right|$-components, but not between $\left|E_{x}\right|$ and $\left|E_{y}\right|$. A separate color scale is used for the experimental results. The two-dimensional distribution of the amplitude of the field is similar for the experiment and for the three calculated cases. The $\left|E_{x}\right|$ component is situated inside the hole and resembles an oblong shape that is elongated in the direction of the incident polarization; the $\left|E_{y}\right|$ component has four lobes, localized at the four "corners" of the hole; the $\left|E_{z}\right|$ component is strongly localized near the edges of the hole in the direction of the incident field. Surprisingly, the two-dimensional field distributions shown in Fig. 4 not only agree very well with the measured near-field distributions, but they also look very much alike.

The main difference lies in the amplitude of the field as we noted previously. The "In contact" and "In contact and filled" cases have similar amplitude levels. In comparison, the "Free standing" case is almost twice as weak. A comparison with Fig. 2 shows that this is explained by the fact that $300 \mathrm{GHz}$ corresponds to a frequency which is much lower than the cut-off frequency of the hole in a free standing metal $(1.4 \mathrm{THz})$, when compared to a hole in metal on $\mathrm{GaP}$ and filled with $\mathrm{GaP}(0.5 \mathrm{THz})$, as shown in Fig. 1. Similar results were obtained at other frequencies below the lowest cut-off frequency of the holes, that is, below $0.5 \mathrm{THz}$. Therefore, we conclude that below the cut-off frequency, for such a thin metal layer, the presence of the $\mathrm{GaP}$ crystal does not perturb the two-dimensional spatial distribution of the field very much. However, the overall spectral response recorded beneath the hole is certainly very different.

\section{Thick metal layer: influence of the GaP crystal}

\subsection{Frequency dependence}

To study the effect of the metal layer thickness on the electric near-field of the hole, we have performed measurements and calculations for a hole defined in a $200 \mu \mathrm{m}$ thick metal foil. Four configurations were studied, shown schematically in Fig. 5. In all cases, a $150 \mu \mathrm{m}$ diameter hole is defined in the metal, which is assumed to be a perfect conductor. The incident field is linearly polarised along the $x$-axis and propagates from top to bottom. In the first case, "Free standing", the foil is placed in vacuum. In the second case, "In contact", a GaP crystal is placed underneath -in contact- with the metal layer. In the third case, "In contact and filled", the GaP crystal is also placed below and in contact with the metal, but now the hole is also filled with

\#114714 - \$15.00 USD Received 23 Jul 2009; revised 11 Sep 2009; accepted 11 Sep 2009; published 15 Sep 2009

(C) 2009 OSA

28 September 2009 / Vol. 17, No. 20 / OPTICS EXPRESS 17417 
$\left|E_{x}\right|$

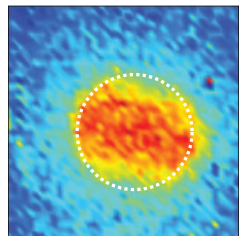

"Measurement"

$10 \mu \mathrm{m} \hat{\mathrm{Au}} \square$

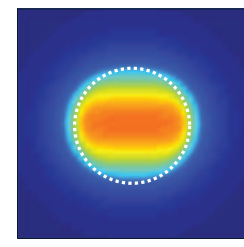

"In contact"

$10 \mu \mathrm{m}$
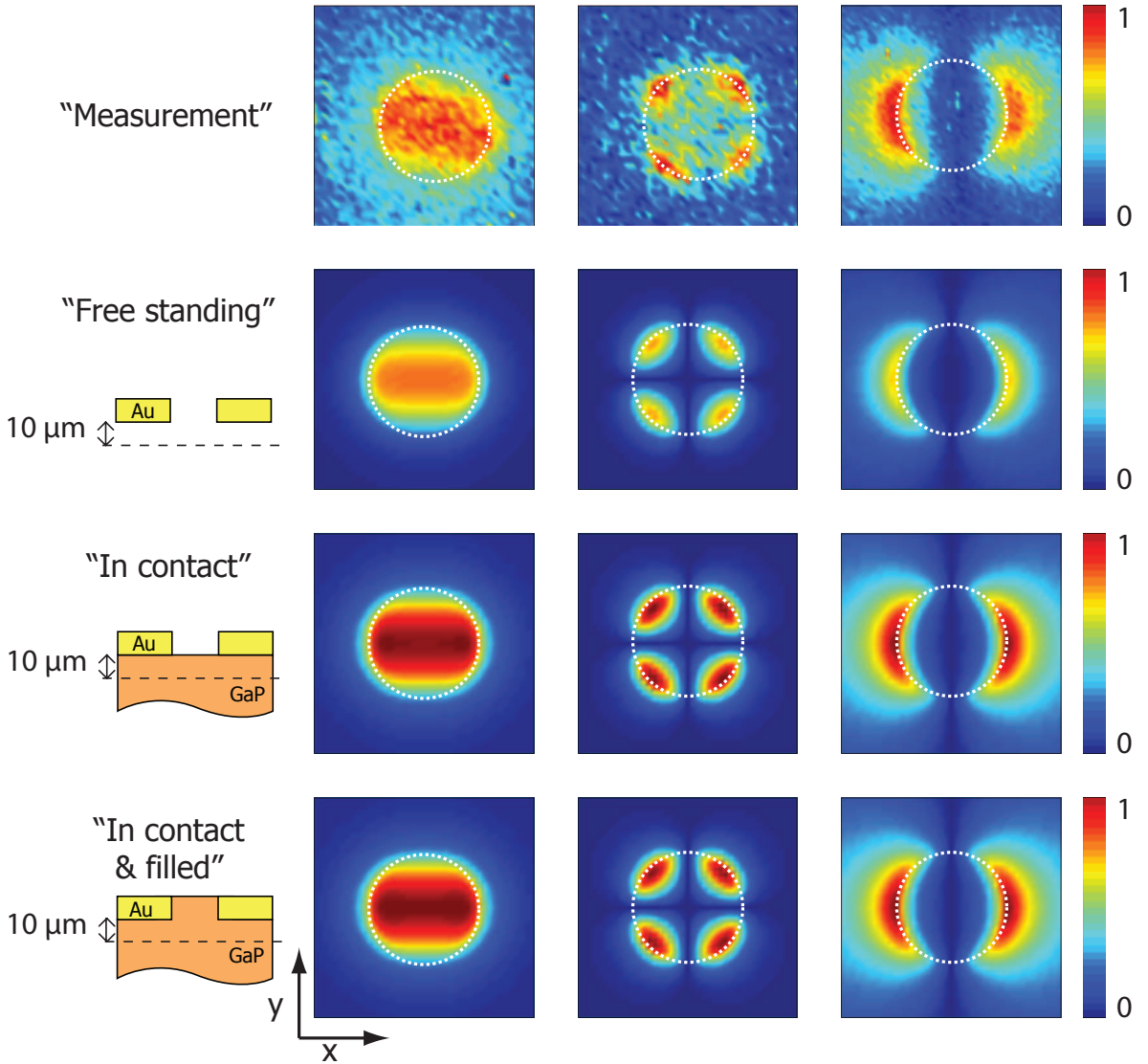

Fig. 4. Spatial distribution of the electric near-field components, $\left|E_{x}\right|,\left|E_{y}\right|$ and $\left|E_{z}\right|$, below a $100 \mu \mathrm{m}$ circular hole at $300 \mathrm{GHz}$ in the $x-y$ plane; top row: measurements taken beneath a $200 \mathrm{~nm}$ thick gold layer; lower rows: calculations using a $0.5 \mu \mathrm{m}$ thick gold layer. The size of the figures is $200 \mu \mathrm{m}$ by $200 \mu \mathrm{m}$. The distance to the metal at which these distributions are calculated is indicated on the left. The incident THz field is polarized along the $x$-axis. The dotted white line represents the position of the hole. For the calculations, the color scales are the same for each component separately. Different color scales were used for the measured field components. 
GaP. In these three cases, the electric field is calculated $10 \mu \mathrm{m}$ below the metal layer. Finally, in the last case, "30 $\mu$ m air gap", the GaP crystal is placed $30 \mu \mathrm{m}$ away from the metal layer and the electric field is calculated $10 \mu \mathrm{m}$ below the surface of the GaP crystal. This last case is studied because it most closely resembles the experiment; it is probable that there is an air gap between the foil and the GaP crystal due to the non-flatness of the sample.

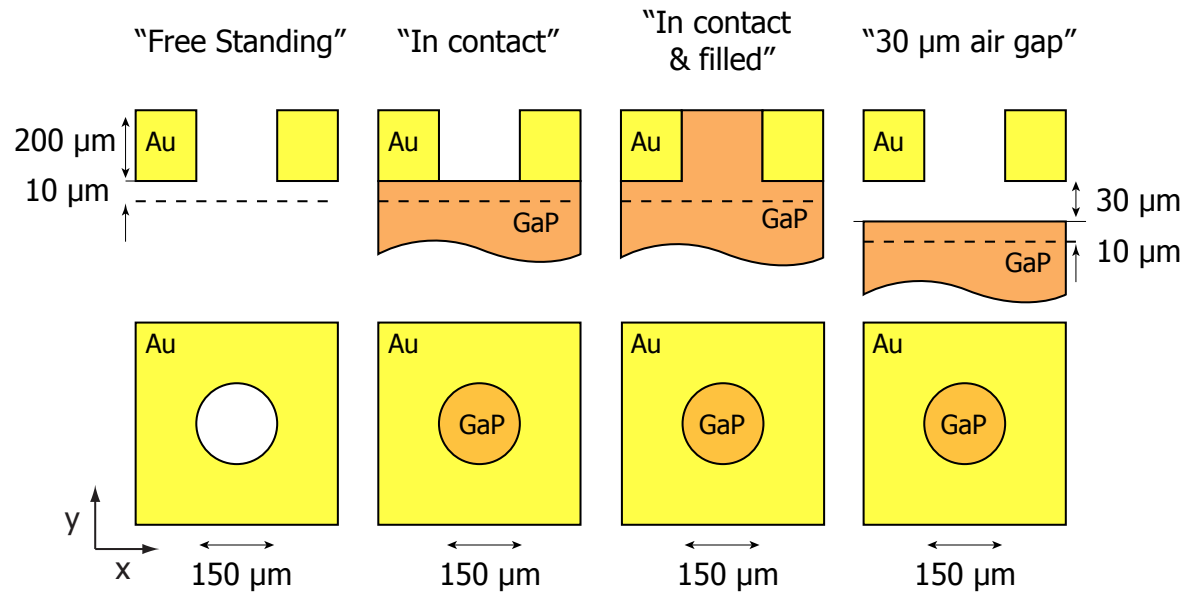

Fig. 5. Drawing of the different configurations used to calculate the electric field beneath a hole in a thick metal layer. The terahertz pulse is incident from above the metal and polarised along the $x$-axis. In the four cases, the circular hole has a diameter of $150 \mu \mathrm{m}$, the thickness of the gold layer, which in the calculations is assumed to be a perfect conductor, is $200 \mu \mathrm{m}$. All the calculated results have been taken $10 \mu \mathrm{m}$ below the metal layer except for the "30 $\mu$ m air gap" for which the total distance to the metal was $40 \mu \mathrm{m}$.

The measurement was performed on a $150 \mu \mathrm{m}$ diameter hole made in a $200 \mu \mathrm{m}$ thick aluminum foil. Due to the relatively large thickness, it was impossible to sputter the metal on the crystal directly: the foil lies on the crystal, creating a possible gap between the crystal surface and the foil. The terahertz path was purged with dry nitrogen gas, reducing absorption of $\mathrm{THz}$ light by water vapor.

Figures 6 (a)-(b) show the frequency-dependent electric near-field components, $\left|E_{x}\right|$ and $\left|E_{z}\right|$, measured beneath the hole. The error bars reflect the variation in signal strength observed at several points close to each other. For increasing frequency, $\left|E_{x}\right|$ remains very low until a frequency of about $1.1 \mathrm{THz}$ is reached, after which the amplitude increases sharply. A similar behavior is observed for $\left|E_{z}\right|$. This rapid increase of the amplitude for increasing frequencies contrasts with the results shown in Fig. 2 for thin metal layers, where this increase was much more gradual.

In Figs. 6 (c)-(d) we plot the calculated electric near-field, $\left|E_{x}\right|$, underneath the middle of the hole, and $\left|E_{z}\right|$ underneath the edge of the hole, in the four different configurations shown in Fig. 5. In all four cases, the hole acts like a high-pass filter: the transmission sharply increases for increasing frequencies, followed by a slow decay and/or a plateau. The "cut-off" frequencies for the "In contact and filled" case are $350 \mathrm{GHz}$ and around $1.2 \mathrm{THz}$ for all the other cases. Note that the maximum amplitudes of the fields, calculated by taking the presence of the crystal into account, are weaker than for the "Free standing" case. This is presumably due to the crystal which causes an increased reflection and a mode mismatch at the output of the hole.

In general, the oscillations present in the simulated "In contact \& filled" case originate from

\#114714 - \$15.00 USD Received 23 Jul 2009; revised 11 Sep 2009; accepted 11 Sep 2009; published 15 Sep 2009

(C) 2009 OSA

28 September 2009 / Vol. 17, No. 20 / OPTICS EXPRESS 17419 

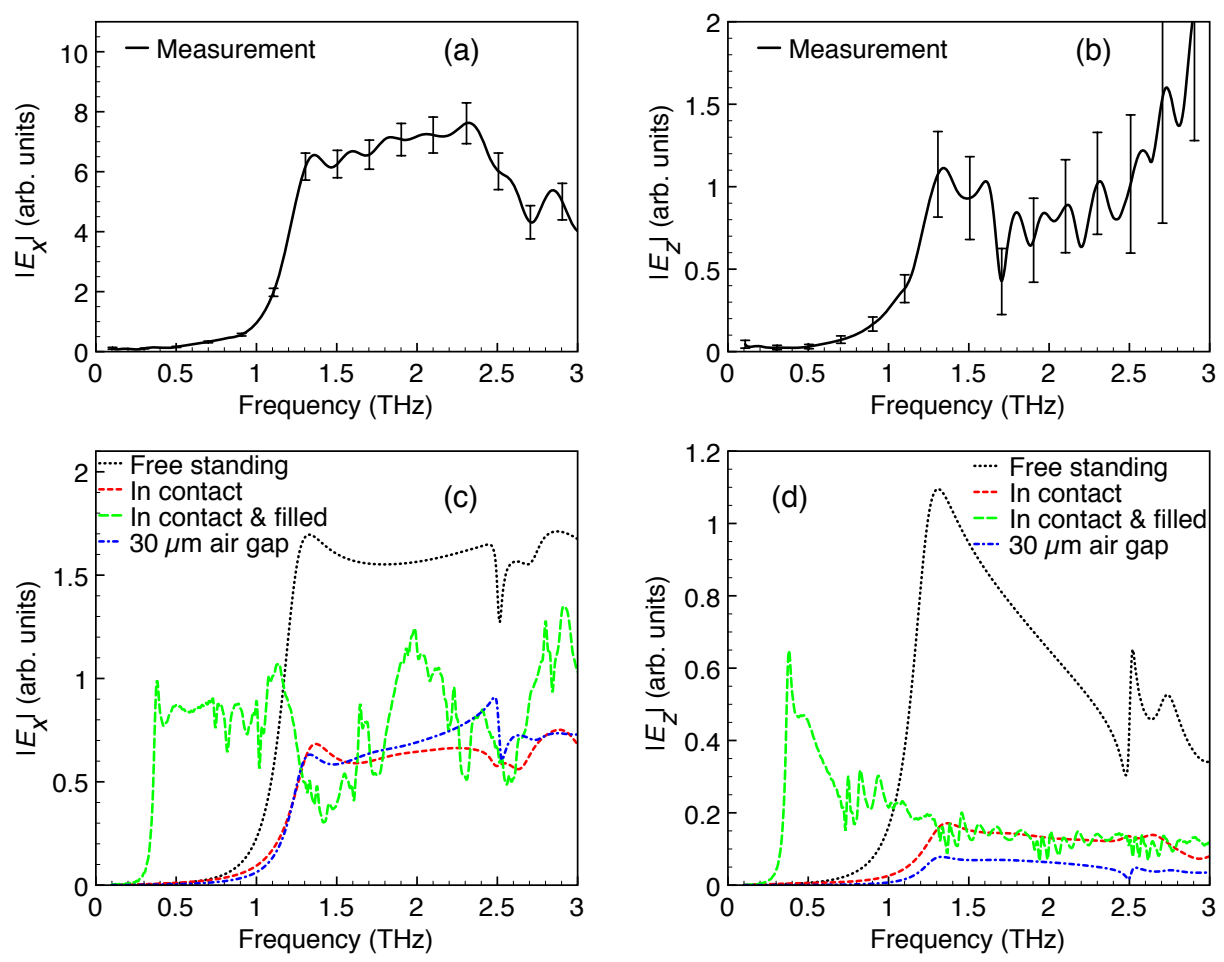

Fig. 6. a) Electric near-fields $\left|E_{x}\right|$ and b) $\left|E_{z}\right|$ as a function of frequency, measured beneath a $150 \mu \mathrm{m}$ hole in a $200 \mu \mathrm{m}$ thick aluminum foil lying on top of a GaP crystal. The curves in a) and b) are guides to the eye. Electric near-fields c) $\left|E_{x}\right|$ and d) $\left|E_{z}\right|$ as a function of frequency, calculated below a $150 \mu \mathrm{m}$ diameter hole defined in a $200 \mu \mathrm{m}$ thick metallic foil, for four different configurations shown in Fig. 5. All near-field spectra are normalized with respect to the spectrum of the incident pulse. Vertical scales of the measurements and the calculations should not be compared directly.

the combination of allowed waveguide modes that exist in the waveguide and the different Fabry-Perot interferences that a short waveguide can produce. The calculations clearly show that for thick metal foils, the frequency-dependent transmission for filled and unfilled holes is very different, which contrasts sharply with the results for the thin metal layer. The experimental results for the thick metal foil also don't agree with those of the "In contact and filled" case. In fact, the experimental results more closely resemble the "In contact" and the "30 $\mu \mathrm{m}$ air gap" cases. The agreement with the "Free standing" case is slightly worse but cannot totally be excluded. Note that in this case, the measured cut-off frequency of about $1.2 \mathrm{THz}$, but also the calculated cut-off frequencies for the "In contact" and "30 $\mu$ m air gap" cases, are very close to the calculated cut-off frequency of the free standing foil. Apparently, therefore, a relatively small gap of only a fraction of a wavelength between the foil and the GaP crystal, is sufficient to dramatically reduce the effect of the crystal on the frequency-dependent transmission of the hole. The proximity of the crystal does, however, lead to changes in the calculated maximum amplitude of the electric near-field beneath the hole. 


\subsection{Metal layer thickness dependence}

So far, we have shown results for two extreme cases: one in which the metal layer is very thin $(0.5 \mu \mathrm{m})$ and one in which the metal is thick $(200 \mu \mathrm{m})$. In Figs. 7 (a) and (b) we plot the frequency-dependent electric near-field components, $\left|E_{x}\right|$ and $\left|E_{z}\right|$, calculated beneath a $100 \mu \mathrm{m}$ diameter hole for four different metal thicknesses: $0.5,2,50$ and $200 \mu \mathrm{m}$. The near-field spectra are normalized with respect to the input spectrum of the incident pulse used in the calculations. In all figures, $\left|E_{x}\right|$ is calculated underneath the center of the hole and $\left|E_{z}\right|$ underneath the edge.

In the "Free standing" case, both $\left|E_{x}\right|$ and $\left|E_{z}\right|$ increase gradually with increasing frequency for the thin metal layers, but rather sharply for the $200 \mu \mathrm{m}$ thick metal layer at a frequency around $1.75 \mathrm{THz}$. This value agrees with the cut-off frequency of $1.75 \mathrm{THz}$, calculated for the lowest mode present in a circular waveguide, the $\mathrm{TE}_{11}$ mode, using the following expression [16]:

$$
v_{\text {cut }- \text { off }}=\frac{c Q_{n l}}{2 \pi a} \frac{1}{\sqrt{\varepsilon \mu}}
$$

where $\varepsilon$ and $\mu$ are the permittivity and the permeability of the material inside the waveguide, $a$ is the radius of the hole and $Q_{n l}$ is a constant for the $\mathrm{TE}_{n l}$ mode with $Q_{11}$ equal to 1.841 . For thinner metal layers, the calculations plotted in Figs. 7 (a) and (b), show a less well defined "cut-off" frequency. Waves with frequencies below the "cut-off" frequency normally don't propagate inside the waveguide because their wave-vector component $k_{z}$ in the propagation direction is imaginary. The waves are then evanescent and their amplitudes decay exponentially in the waveguide with distance. If the waveguide is shorter than the wavelength, however, these evanescent waves are still able to reach the end of the waveguide and are transferred into propagating waves again. This explains the gradual increase in the electric near-field underneath the holes in the thin metal layers, as opposed to the more abrupt increase observed for the thick metal layer. Note that the differences between metal layers of $2 \mu \mathrm{m}$ and $0.5 \mu \mathrm{m}$ thickness are very small. Below $0.5 \mu \mathrm{m}$, there is no difference in the results obtained by the calculations.

In Figs. 7 (c) and (d), we plot the electric fields, $\left|E_{x}\right|$ and $\left|E_{z}\right|$, as a function of frequency, calculated in a plane $10 \mu \mathrm{m}$ below the hole, for metal layer thicknesses of $0.5,2,10$ and $25 \mu \mathrm{m}$. In this case the metal is assumed to be in contact with the GaP crystal. In Figs. 7 (e) and (f) similar calculations are shown, but this time the hole is also assumed to be filled with GaP. Again, the electric near-field spectrum is normalized with respect to the incident field spectrum. The curves for a metal thickness of $0.5 \mu \mathrm{m}$ are the same as the ones shown in Fig. 2 and are reproduced here for completeness. In the "In contact" case, the frequency-dependence of both $\left|E_{x}\right|$ and $\left|E_{z}\right|$ is strongly affected by the metal thickness, where significant changes already occur when the thickness increases from 0.5 to $10 \mu \mathrm{m}$. Note that $10 \mu \mathrm{m}$ is still a factor of ten smaller than the diameter of the hole.

Both in the "In contact" and "In contact and filled" cases, we can see an increase of the field amplitude for $\left|E_{x}\right|$ with frequency until a maximum or a quasi-plateau is reached, after which the amplitude decays again. The initial, almost linear, dependence of the amplitude with frequency agrees with the linear dependence predicted by Bouwkamp [10] and also with experiments reported recently.[9]

In the "In contact" case, the cut-off frequency clearly shifts to higher frequencies with increasing metal layer thickness. This is explained by the fact that for waveguides of increasing length, the influence of the substrate at the end of the waveguide diminishes, so that the behavior of the waveguide tends to approach that of a free standing waveguide. Note that Figs. 7 (c) and (d) only show results for waveguides as long as $25 \mu \mathrm{m}$ and thus only show the onset of this behavior. In contrast, when the hole is also filled, the cut-off frequency does not change much with increasing metal layer thickness; only the steepness of the curve in the case of $\left|E_{x}\right|$ increases somewhat with larger thickness. If we consider the hole as an ideal circular

\#114714 - \$15.00 USD Received 23 Jul 2009; revised 11 Sep 2009; accepted 11 Sep 2009; published 15 Sep 2009 

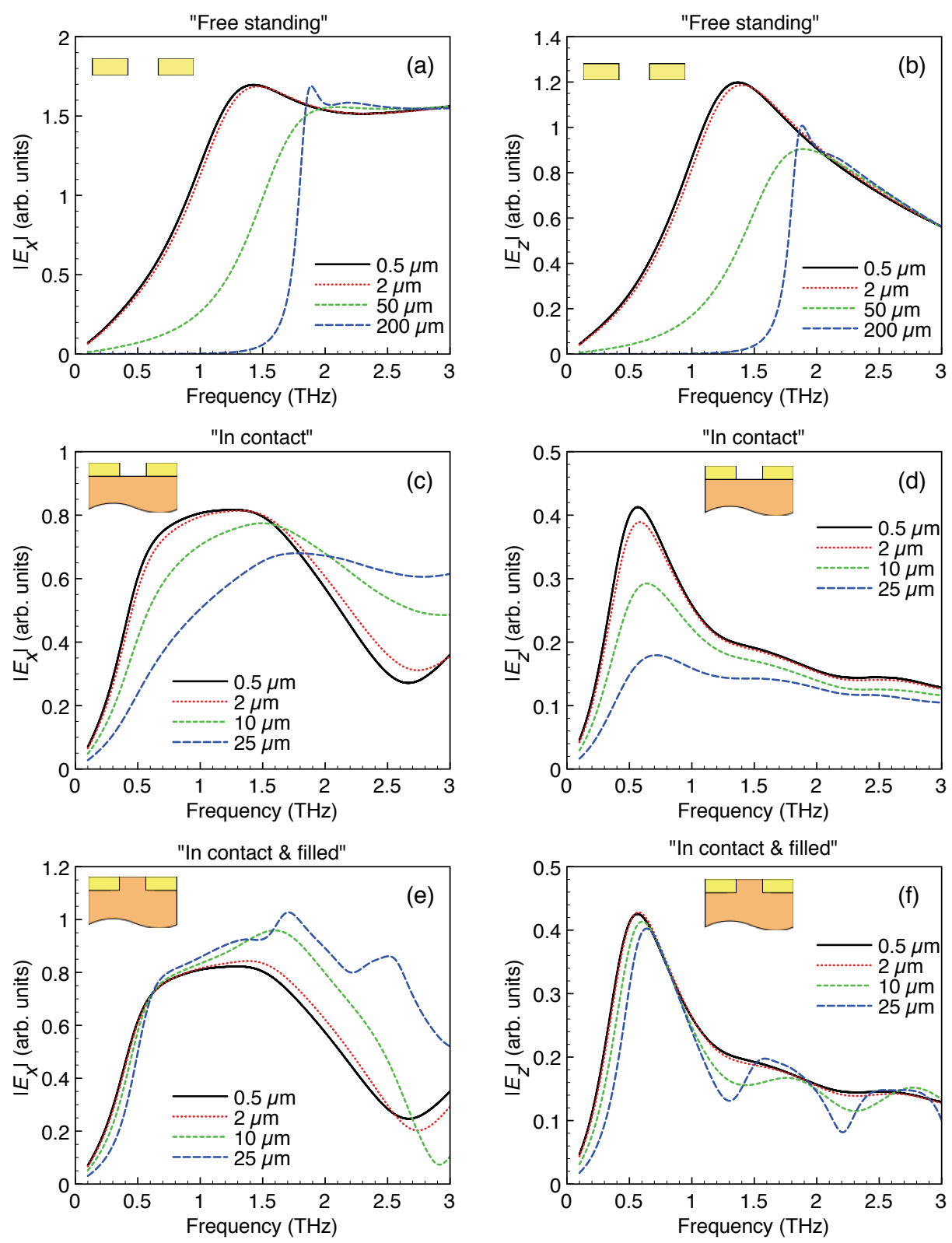

Fig. 7. Electric near-field components $\left|E_{x}\right|$ (left) under the center of a $100 \mu \mathrm{m}$ diameter hole and $\left|E_{z}\right|$ (right) underneath the edge of the hole, calculated as a function of frequency in a plane that is $10 \mu \mathrm{m}$ below the metal layer. a) and b): the "Free standing" case, for four different metal layer thicknesses: 0.5, 2, 50 and $200 \mu \mathrm{m} ; \mathrm{c}$ ) and d): the GaP "In contact"; e) and f): the GaP "In contact and filled" cases. The latter two cases are plotted for four different metal layer thicknesses of $0.5,2,10$ and $25 \mu \mathrm{m}$.

\#114714 - \$15.00 USD Received 23 Jul 2009; revised 11 Sep 2009; accepted 11 Sep 2009; published 15 Sep 2009 (C) 2009 OSA 28 September 2009 / Vol. 17, No. 20 / OPTICS EXPRESS 17422 
waveguide, filled with GaP having a refractive index of 3.4, we obtain a cut-off frequency of $586 \mathrm{GHz}$. The calculation shown in Fig. 7 gives a "cut-off" frequency around $540 \mathrm{GHz}$ for a thickness of $25 \mu \mathrm{m}$. Therefore, when the hole is in contact with and filled with GaP, the "cut-off" frequency is close that of a waveguide entirely embedded in GaP. An important conclusion can be drawn from the calculations and measurements of the frequency-dependent hole transmission in metal layers deposited on the GaP crystal: if the hole is completely filled with GaP, the transmission characteristics are very similar to those of an empty hole, but only when the metal layer is thin enough. For the hole diameter of $100 \mu \mathrm{m}$ used in the calculations, this approximation already breaks down for metal layer thicknesses larger than 5-10 $\mu \mathrm{m}$. Note that this agrees with our earlier observations of the frequency-dependent component, $\left|E_{z}\right|$, measured beneath holes fabricated in a $200 \mathrm{~nm}$ thick gold layer deposited on GaP.[9]

\subsection{Near-field distribution}

In Fig. 8, we plot the two-dimensional spatial distribution of the amplitude of the three orthogonal field components, $\left|E_{x}\right|,\left|E_{y}\right|$, and $\left|E_{z}\right|$, of the electric near-field in a plane underneath a $200 \mu \mathrm{m}$ thick metal layer at a frequency of $1 \mathrm{THz}$. At this frequency, the hole can still be considered as sub-wavelength since $1 \mathrm{THz}$ is below the cut-off frequency of the waveguide. The top row shows the actual measurements, whereas the images in the remaining rows represent calculations for the various configurations shown on the left: a plot of the field components calculated in a plane $10 \mu \mathrm{m}$ below the metal (second row), $40 \mu \mathrm{m}$ below the metal (third row), $10 \mu \mathrm{m}$ below the metal which is in contact with the GaP (fourth row), $40 \mu \mathrm{m}$ below the metal which is in contact with the GaP (fifth row) and finally, $10 \mu \mathrm{m}$ below the surface of the GaP crystal, where the surface is $30 \mu \mathrm{m}$ below the metal (sixth row). This last configuration most likely resembles the condition under which the experiment was performed. All calculations use the same color scale for each field component separately: the amplitudes calculated for the different configurations can therefore be compared directly.

The calculated spatial distribution of the electric near-field at $10 \mu \mathrm{m}$ is somewhat different from the distribution calculated in a plane $40 \mu \mathrm{m}$ below the metal. For example, at a distance of $40 \mu \mathrm{m},\left|E_{z}\right|$ is less concentrated at the edges of the hole and $\left|E_{y}\right|$ shows significant presence underneath the metal. The amplitude of $\left|E_{z}\right| 40 \mu \mathrm{m}$ below the metal is also significantly lower than at $10 \mu \mathrm{m}$ below the layer. This is to be expected, since the $z$-component is non-propagating and decays rapidly with the distance to the metal layer.

For the "Free standing" cases as well as the "In contact" cases, $\left|E_{x}\right|$ and $\left|E_{y}\right|$ are present mostly below the hole. The "30 $\mu \mathrm{m}$ air gap" case is noteworthy in that, $\left|E_{y}\right|$ spreads out to regions beyond the hole, underneath the metal, becoming more delocalized. The presence of a dielectric held at a certain distance from the metal distorts the field significantly compared to the "Free standing" case and the "In contact" cases. This can be understood from the refraction of the quasi-static field lines at the air/crystal interface. Although difficult to see in Fig. 8, $\left|E_{z}\right|$ in the lower three rows is mainly present near the edges of the hole, as the Bouwkamp model predicts.[10] An important observation is the value of $\left|E_{z}\right|$, which for the "30 $\mu$ m air gap" case is 20 times lower than for the "Free standing" case and 4 times lower than the "In contact" case. This explains our experimental observation that even small gaps between the metal foil and the crystal give rise to a strongly reduced signal, making it more difficult to measure weak field components such as the $y$-component of the field for a square aperture for example.[17].

Our measurements agree mostly with the "30 $\mu \mathrm{m}$ air gap" calculation. The measured $y$-component is more delocalized than expected when compared with the "In contact" calculation. At the same time, the measured $z$-component is weak but still localized around the edges and agrees very well with these calculations.

\#114714 - \$15.00 USD Received 23 Jul 2009; revised 11 Sep 2009; accepted 11 Sep 2009; published 15 Sep 2009

(C) 2009 OSA

28 September 2009 / Vol. 17, No. 20 / OPTICS EXPRESS 17423 


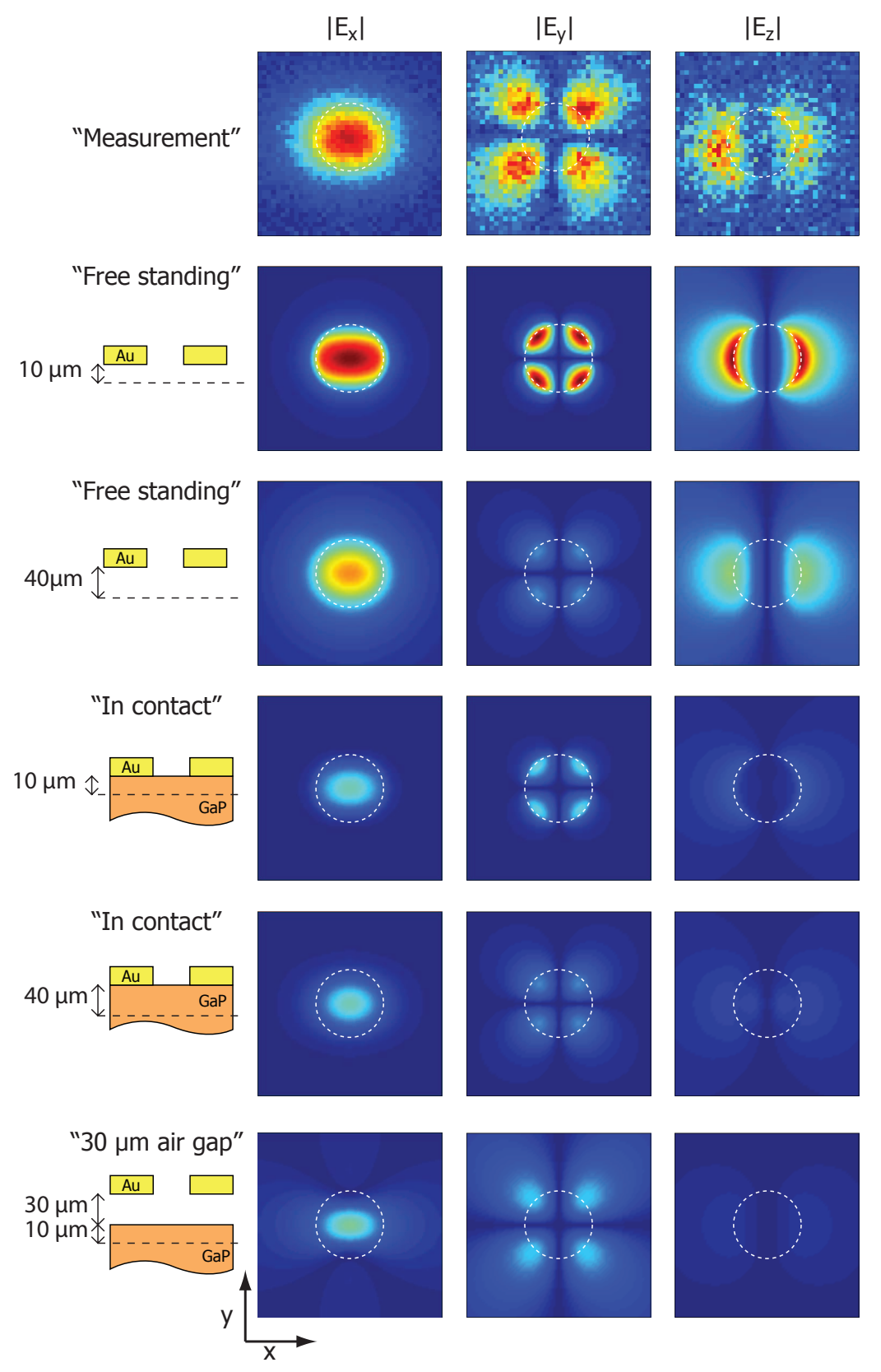

Fig. 8. Spatial distribution of the electric near-field components, $\left|E_{x}\right|,\left|E_{y}\right|$ and $\left|E_{z}\right|$, underneath a $150 \mu \mathrm{m}$ circular hole in a $200 \mu \mathrm{m}$ thick plate at $1 \mathrm{THz}$ in the $x-y$ plane. Top row: measurements; lower rows: calculations. The size of the figure is $400 \mu \mathrm{m}$ by $\overline{400 \mu \mathrm{m}}$. The distance between this plane and the metal layer is indicated on the left in each case (10 or $40 \mu \mathrm{m})$. The incident THz field is polarized along the $x$-axis. The dotted white line represents the position of the hole. For the calculations, the color scales are the same for each component separately. Different color scales were used for the measured field components. 


\section{Conclusion}

The effect on the electric near-field of the GaP crystal can be summarized as follow: for thin metal layers, the spatial distribution is not altered very much for frequencies below the lowest cut-off frequency obtained for all configurations used in our calculations. At the same time, the transmission properties of a hole filled with $\mathrm{GaP}$ are practically identical to those of an unfilled hole. When the metal layer thickness increases, the frequency-dependent transmission of both filled and unfilled holes changes dramatically and become different from each other. For large thickness, the transmission of the unfilled hole on GaP resembles the one of a hole with no crystal underneath it. The presence of an air gap between the structure and the crystal decreases the amplitude of the detected signal and gives rise to less confined fields due to an increased divergence of the quasi-static field lines. Our results also imply that the presence of a near-field probe in the form of a dielectric substrate, can affect the spectral response of the object under study. This is, of course, true for our near-field measurements as well, although our "probe", the GaP crystal on which the metal is deposited, forms a dielectric half space which is easily included in the analysis.

\section{Acknowledgements}

We gratefully acknowledge financial support from Nederlandse Organisatie voor Wetenschappelijk Onderzoek (NWO) and from the EU TERANOVA Program (RCN-71835). Lucie Guestin's participation in this research was made possible with the support of the European cooperation and mobility program "Erasmus Mundus". Paul Planken gratefully acknowledges support from a NWO-VICI grant. 